\title{
Symbiotic Treatment Improve IBS Symptoms and Quality of Life: Placebo-Controlled Study
}

\section{Bazzi Nagham¹, Bazzi Mariam² ${ }^{2}$ Hallal Hadi' ${ }^{1}$ Lakkis Remie ${ }^{1}$, Akoush Hassan $^{1}$, Saad Wajih ${ }^{1}$, AlKhatib Amani ${ }^{1}$ and Hallal Mahmoud ${ }^{3 *}$}

${ }^{1}$ Faculty of Medicine, Lebanese University, Beirut, Lebanon

${ }^{2}$ Faculty of Pharmacy, Lebanese International University and Faculty of Public

Health, Saint Joseph University and Faculty of Medicine, Lebanese University,

Beirut, Lebanon

${ }^{3}$ Assistant Professor of Medicine, Faculty of Medicine Sciences, Lebanese

University and Department of Gastroenterology, Al Zahraa Hospital University

Medical Center, Beirut, Lebanon

*Corresponding Author: Hallal Mahmoud, Assistant Professor of Medicine, Faculty of Medicine Sciences, Lebanese University and Department of Gastroenterology, $\mathrm{Al}$

Zahraa Hospital University Medical Center, Beirut, Lebanon.
Received: February 22, 2021

Published: March 19, 2021

(C) All rights are reserved by Hallal

Mahmoud., et al.

\begin{abstract}
Background: Irritable bowel syndrome (IBS) is a chronic and functional gastrointestinal dysfunction characterized by altered bowel movement and abdominal pain and has a complicated etiology. Probiotics are the novel therapy based on better understanding of the disease pathology.

Patients and Methods: We conducted a control-placebo study, 157 subjects enrolled in this study, and followed up for 6 months, divided into three groups to compare the effect of probiotics and placebo on IBS patients.

Results: Probiotics significantly decreased the IBS symptoms, it normalized bowel movement.

Conclusion: Probiotics could be the gold standard for IBS treatment.

Keywords: Irritable Bowel Syndrome; Probiotics; Constipation and Diarrhea
\end{abstract}

\section{Introduction}

Irritable bowel syndrome (IBS) is a chronic and functional gastrointestinal dysfunction characterized by altered bowel movement and abdominal pain and has a complicated etiology [1,2]. Prevalence of IBS is estimated between 5 - 11\% in North America and $11.4 \%$ in Saudi Arabia, however there are scarce data on the prevalence of IBS in Middle EAST [3]. IBS age of onset is at 20 to 39 years age old [3]. Female sex, food intolerance, stress, psychological problems are risks factors [4]. Nuances of clinical presentation are abdominal pain or discomfort, stool pattern alteration, bowel distension, bloating, and urgency [5]. The IBS can be classed into three groups: IBS-D (diarrhea dominant), IBS-C (constipation dominant), and IBS-M (mixed bowel patterns) [4]. The disorder can be psychologically devastating and depressing [6]. Anxiety and depression are three times more common in IBS patients compared to healthy patients [7]. It is also challenged by increasing socioeconomic costs due to earlier age risk [5].

Nowadays, there has been heightened interest in using symbiotic combinations as a treatment modality for IBS. The latter refers to a new term that represents the association between combinations of a probiotic and prebiotic [8]. These microorganism are capable of relieving IBS symptoms and hold a promise of new therapeutic strategy [5]. They were initially described in 1908 by 
ElieMetchinkoff who noticed the beneficial effect of fermented food on human health [9]. The symbiotic microorganism have been proved to prevent pathogenic bacteria growth and host invasion, improve abdominal pain in animals as well as amelioration of IBS symptoms [9].

\section{Aim of the Study}

The aim of this clinical trial was to evaluate the effectiveness of a symbiotic product, Lactobacillus acidophilus + Lactobacillus Bifidus + sunfiber (Vita Colon Relief ${ }^{\circledR}$ ) for IBS-related symptoms, in the improvement of IBS symptoms: abdominal pain score, bloating score, Quality of Life (QOL) score, and number of bowel movement in healthy, IBS-C, IBS-D and IBS-M patients.

\section{Methods}

This is a prospective, randomised, placebo-controlled study.

The protocol was approved by an independent IRB obtained from a hospital University.

Subjects aged 18 years or older were recruited in the study. Included subjects had to meet the Rome IV criteria for IBS [10]. This criteria include presence of recurrent abdominal pain or discomfort at least 1 day/week in the last 3 months, associated with 2 or more of the following: related to defecation defecation, onset associated with a change in frequency of stool, and onset associated with a change in form (appearance) of stool. This criteria is fulfilled with symptoms lasting for 6 months [10], 157 subjects were enrolled and classed into three groups:

1. Group 1 patients taking Vita Colon Relief ${ }^{\circledR}$

2. Group 2 patient taking Vita Colon Relief ${ }^{\circledR}+$ other drug (not related to colon disease)

3. Group 3 patient taking placebo (control).

The Vita Colon Relief ${ }^{\circledR}$ consists of a smooth tablet containing: $28.056 \mathrm{mg}$ Lactobacillus acidophilus, Lactobacillus bifidus (33.4 $\mathrm{mg}$ ), and Sunfiber ${ }^{\circledR}$ (500 mg).

Subjects returned to the clinic at 1 month, 3 months and at 6 months to answer a clinical hard copy survey which consists of a Numeric Pain Scale to assess:

- Abdominal pain (0: no pain, 1 - 3: mild pain, 4 - 6: moderate pain, 7 - 10: severe pain)

- Bloating score with 5 answer options graded from 1 to 5 ,

- Quality of life improvement with 4 answer options: $25 \%$, $50 \%, 75 \%$ and $100 \%$,
- An open question about the number of bowel movement per week including number of diarrhea or constipation per week [11].

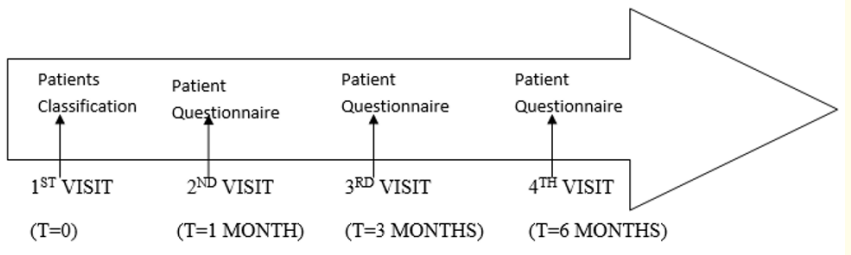

Figure

\section{Results}

Study endpoints

Study endpoints include change in abdominal pain score, bloating and distension score, quality of life improvement, change in bowel movement and frequency of stool per week. Safety endpoints were the severity and exacerbation of IBS. Patients having severe adverse events were excluded.

\section{Data management}

Data were collected on hard-copy surveys in the clinic and then entered into excel program. Data were analyzed using SPSS program and inter-group variation were assessed.

\section{Demographics and baseline subject characteristics}

The distribution of demographic and baseline characteristics of the population are presented in table 1 . The three groups are comparable in age and sex. The most common chief complaint in all groups was abdominal pain. The second most common symptom was abdominal distention and bloating in group 1 , abdominal bloating in group 2 and abdominal distention in group 3 .

\section{Distribution of irritable bowel syndrome subtypes}

Among the 157 patients, 109 were classified by the investigators as IBS-C, IBS-D, or IBS-M based on their symptoms and history at study entry. The distribution of subjects in the three subtypes was presented in table 1 . In the three groups the most common subtype was IBS-M.

\section{Abdominal pain score}

A significant difference was noted on visit 3 (at 4 months) in abdominal pain score between the three groups. At the $4^{\text {th }}$ visit (longest follow up, 6 months), most patients (46.2\%) in group 1 had 


\begin{tabular}{|c|c|c|c|c|c|}
\hline & & Vita Colon & $\begin{array}{l}\text { Vita Colon+ } \\
\text { Other Drug }\end{array}$ & Placebo & Significant \\
\hline Total (n) & & 54 & 54 & 49 & \\
\hline \multirow{2}{*}{ Age (years) } & Mean & 44.2 & 43.46 & 45.45 & \multirow{2}{*}{0.881 (NS) } \\
\hline & Standard deviation & 13.331 & 13.212 & 49 & \\
\hline \multirow{2}{*}{ SEX (\#, \%) } & Male & $23(42.3 \%)$ & $22(40.7 \%)$ & $16(32.6 \%)$ & \multirow{2}{*}{$0.551(\mathrm{NS})$} \\
\hline & Female & $31(57 \%)$ & $32(59.2 \%)$ & $33(67.3 \%)$ & \\
\hline \multirow{6}{*}{$\begin{array}{l}\text { Chief com- } \\
\text { plaint }(\#, \%)\end{array}$} & Abdominal pain & $23(42 \%)$ & $34(62.9 \%)$ & $21(42.8 \%)$ & \multirow{6}{*}{0.007} \\
\hline & Abdominal bloating & $13(24 \%)$ & $13(29.6 \%)$ & $7(14.2 \%)$ & \\
\hline & Flatulence & $8(14.8 \%)$ & $2(3.7 \%)$ & $9(18.3 \%)$ & \\
\hline & Diarrhea & $8(14.8 \%)$ & $9(16.6 \%)$ & $1(2 \%)$ & \\
\hline & constipation & $8(14.8 \%)$ & $13(24 \%)$ & $9(18.3 \%)$ & \\
\hline & distention & $13(24 \%)$ & $14(25.9 \%)$ & $19(38.7 \%)$ & \\
\hline \multirow{3}{*}{$\begin{array}{l}\text { IBS type (\#, } \\
\%)\end{array}$} & IBS-C & $6(11 \%)$ & $12(22.2 \%)$ & 0 & \multirow{3}{*}{0.000} \\
\hline & IBS-D & $4(7.4 \%)$ & $1(1 \%)$ & 0 & \\
\hline & IBS-M & $27(50 \%)$ & $18(33.3 \%)$ & $41(83.6 \%)$ & \\
\hline
\end{tabular}

Table 1: Patients description.

NS: not significant: $\mathrm{p}>0.05$.

no abdominal pain versus only $11.1 \%$ in visit 1 and none scored more than 3 in abdominal pain score during the third and fourth visit. However $9.2 \%$ in group 2 and $2 \%$ in group 3 (placebo) scored between 4 and 6 in the third visit and $7.4 \%$ in group 2 and $2 \%$ in group 3 (placebo) scored between 4 and 6 in the fourth visit.

\begin{tabular}{|c|c|c|c|c|c|c|}
\hline & & & Vita Colon & $\begin{array}{l}\text { Vita Colon+ } \\
\text { Other Drug }\end{array}$ & Placebo & Significant? \\
\hline \multirow{16}{*}{$\begin{array}{l}\text { Abdominal } \\
\text { Pain score }\end{array}$} & \multirow{4}{*}{$\mathrm{V} 1$} & 0 & $6(11.1 \%)$ & $5(9.2 \%)$ & $0(0 \%)$ & \multirow{4}{*}{0.043 (NS) } \\
\hline & & $1-3$ & $17(31.5 \%)$ & $8(14.8 \%)$ & $10(20.4 \%)$ & \\
\hline & & $4-6$ & 28 (51.8\%) & $34(62.9 \%)$ & 37 (75.5\%) & \\
\hline & & $7-10$ & $3(5.5 \%)$ & 7 (12.9\%) & $2(4 \%)$ & \\
\hline & \multirow{4}{*}{$\mathrm{V} 2$} & 0 & $9(16.6 \%)$ & $15(27.7 \%)$ & $1(2 \%)$ & \multirow{4}{*}{0.006} \\
\hline & & $1-3$ & $38(70.3 \%)$ & $30(55.5 \%)$ & $42(85.7 \%)$ & \\
\hline & & $4-6$ & 7 (12.9\%) & $9(16.6 \%)$ & $6(12.2 \%)$ & \\
\hline & & $7-10$ & 0 & 0 & 0 & \\
\hline & \multirow{4}{*}{ V3 } & 0 & $24(44.4 \%)$ & $32(59.2 \%)$ & $20(40.8 \%)$ & \multirow{4}{*}{0.032} \\
\hline & & $1-3$ & $30(55.5 \%)$ & 17 (31.5\%) & $28(57.1 \%)$ & \\
\hline & & $4-6$ & 0 & $5(9.2 \%)$ & $1(2 \%)$ & \\
\hline & & $7-10$ & 0 & 0 & 0 & \\
\hline & \multirow{4}{*}{ V4 } & 0 & $25(46.2 \%)$ & $30(55.5 \%)$ & $29(59.1 \%)$ & \multirow{4}{*}{$0.078(\mathrm{NS})$} \\
\hline & & $1-3$ & $24(44.44 \%)$ & $13(24.0 \%)$ & $17(34.6 \%)$ & \\
\hline & & $4-6$ & 0 & $4(7.4 \%)$ & $1(2 \%)$ & \\
\hline & & $7-10$ & 0 & 0 & 0 & \\
\hline
\end{tabular}

Table 2: Abdominal pain score.

NS: Not Significant: $\mathrm{p}>0.05$.

\section{Bloating score}

During the four visits, no significant difference were seen between the three groups. In the Vita Colon Relief ${ }^{\circledR}$ group, no bloating score of 4 was detected in the third visit or fourth visit, versus $7.4 \%$ in the first and second visit. However, in the second group, 5.5\% (n = 3) had a bloating score of 4 in the fourth visit and $4 \%(n=2)$ in the placebo group. 


\begin{tabular}{|c|c|c|c|c|c|c|}
\hline & & & Vita Colon & $\begin{array}{l}\text { Vita Colon+ } \\
\text { Other Drug }\end{array}$ & Placebo & Significant? \\
\hline \multirow{16}{*}{$\begin{array}{l}\text { Bloating } \\
\text { score }\end{array}$} & \multirow{4}{*}{ V1 } & 1 & $1(1.8 \%)$ & $5(9.2 \%)$ & $2(4 \%)$ & \multirow{4}{*}{$0.2(\mathrm{NS})$} \\
\hline & & 2 & $19(35.15 \%)$ & $15(27.7 \%)$ & $19(38.7 \%)$ & \\
\hline & & 3 & $30(55.5 \%)$ & $23(42.5 \%)$ & $22(34.8 \%)$ & \\
\hline & & 4 & $4(7.4 \%)$ & $11(20.3 \%)$ & $6(12.2 \%)$ & \\
\hline & \multirow{4}{*}{ V2 } & 1 & $3(5.5 \%)$ & 7 (12.9\%) & $3(6.1 \%)$ & \multirow{4}{*}{$0.548(\mathrm{NS})$} \\
\hline & & 2 & $23(42.5 \%)$ & $23(42.5 \%)$ & $23(46.9 \%)$ & \\
\hline & & 3 & $24(44.4 \%)$ & $18(33.3 \%)$ & $21(42.8 \%)$ & \\
\hline & & 4 & $4(7.4 \%)$ & $6(11.1 \%)$ & $2(4 \%)$ & \\
\hline & \multirow{4}{*}{ V3 } & 1 & $16(24 \%)$ & $20(37 \%)$ & $15(30.6 \%)$ & \multirow{4}{*}{0.625 (NS) } \\
\hline & & 2 & $33(51.1 \%)$ & $25(46.2 \%)$ & $29(59.1 \%)$ & \\
\hline & & 3 & $5(9.2 \%)$ & 7 (12.9\%) & $4(8.1 \%)$ & \\
\hline & & 4 & 0 & $2(3.7 \%)$ & $1(2 \%)$ & \\
\hline & \multirow{4}{*}{ V4 } & 1 & $23(42.5 \%)$ & 27 (50\%) & 17 (34.6\%) & \multirow{4}{*}{0.238 (NS) } \\
\hline & & 2 & $23(42.5 \%)$ & $21(38.8 \%)$ & $26(53 \%)$ & \\
\hline & & 3 & $8(14.8 \%)$ & $3(5.5 \%)$ & $4(8.1 \%)$ & \\
\hline & & 4 & 0 & $3(5.5 \%)$ & $2(4 \%)$ & \\
\hline
\end{tabular}

Table 3: Bloating score.

NS: Not Significant: $\mathrm{p}>0.05$.

Quality of life (QOL) improvement

A significant difference was noted between the three groups in the fourth visit regarding the QOL improvement. A 100\% improve- ment was seen in $18.5 \%(n=10)$ in the $1^{\text {st }}$ group, versus $11 \%(n=$ $6)$ and $2 \%(n=1)$ in the second and third groups respectively.

\begin{tabular}{|c|c|c|c|c|c|c|}
\hline & & & Vita Colon & $\begin{array}{l}\text { Vita Colon+ } \\
\text { Other Drug }\end{array}$ & Placebo & Significant? \\
\hline \multirow{15}{*}{ QOL Improvement } & \multirow{3}{*}{$\mathrm{V} 1$} & $25 \%$ & $33(61.1 \%)$ & $32(59.3 \%)$ & $28(57.1 \%)$ & \multirow{3}{*}{0.161 (NS) } \\
\hline & & $50 \%$ & $18(33.3 \%)$ & $15(27.8 \%)$ & $21(42.9 \%)$ & \\
\hline & & $75 \%$ & $2(3.7 \%)$ & $3(5.5 \%)$ & 0 & \\
\hline & \multirow{4}{*}{$\mathrm{V} 2$} & $25 \%$ & $11(20.4 \%)$ & $10(18.5 \%)$ & $2(4 \%)$ & \multirow{4}{*}{0.181 (NS) } \\
\hline & & $50 \%$ & $32(59.3 \%)$ & $31(57.4 \%)$ & 35 (71.4\%) & \\
\hline & & $75 \%$ & $10(18.5 \%)$ & $13(24.0 \%)$ & $12(24.4 \%)$ & \\
\hline & & $100 \%$ & $1(1.9 \%)$ & 0 & 0 & \\
\hline & \multirow{4}{*}{ V3 } & $25 \%$ & $1(1.9 \%)$ & $3(5.5 \%)$ & 0 & \multirow{4}{*}{0.219 (NS) } \\
\hline & & $50 \%$ & $17(31.5 \%)$ & $13(24.1 \%)$ & $13(26.5 \%)$ & \\
\hline & & $75 \%$ & $30(55.6 \%)$ & $35(64.8 \%)$ & $35(71.4 \%)$ & \\
\hline & & $100 \%$ & $6(11.1 \%)$ & $3(5.5 \%)$ & $1(2 \%)$ & \\
\hline & \multirow{4}{*}{ V4 } & $25 \%$ & $1(1.9 \%)$ & $5(9.2 \%)$ & 0 & \multirow{4}{*}{0.029} \\
\hline & & $50 \%$ & $8(14.8 \%)$ & $7(12.9 \%)$ & $6(12.2 \%)$ & \\
\hline & & $75 \%$ & $35(64.8 \%)$ & $35(64.8 \%)$ & $42(85.7 \%)$ & \\
\hline & & $100 \%$ & $10(18.5 \%)$ & $6(11.1 \%)$ & $1(2 \%)$ & \\
\hline
\end{tabular}

Table 4: QOL improvement. NS: Not Significant: $\mathrm{p}>0.05$. 


\section{Number of bowel movement (BM)}

No significant difference was seen in the fourth visit and the majority of patients in the three groups had 3 - 7 BM per week.
An improvement of the number of BM was seen in the first group between first visit where $64.8 \%$ had normal BM (score between 3 - 7) versus $94.4 \%$ in the fourth visit.

\begin{tabular}{|c|c|c|c|c|c|c|}
\hline & & & Vita Colon & $\begin{array}{l}\text { Vita Colon+ } \\
\text { Other Drug }\end{array}$ & Placebo & Significant? \\
\hline & & $<3$ & $16(29.6 \%)$ & $22(40.7 \%)$ & $21(42.8 \%)$ & \\
\hline & V1 & $3-7$ & 35 (64.8\%) & $30(55.6 \%)$ & $24(48.9 \%)$ & 0.483 (NS) \\
\hline & & $>7$ & $3(5.5 \%)$ & $2(3.7 \%)$ & $4(8.1 \%)$ & \\
\hline & & $<3$ & $28(51.9 \%)$ & $20(37 \%)$ & $26(53 \%)$ & \\
\hline Number & V2 & $3-7$ & $26(48.1 \%)$ & $34(62.9 \%)$ & $23(46.9 \%)$ & 0.184 (NS) \\
\hline of bowel & & $>7$ & 0 & 0 & 0 & \\
\hline & & $<3$ & $31(57.4 \%)$ & $16(24 \%)$ & $28(57.1 \%)$ & \\
\hline & V3 & $3-7$ & $23(42.6 \%)$ & $38(70.3 \%)$ & $21(48.9 \%)$ & 0.004 \\
\hline & & $>7$ & 0 & 0 & 0 & \\
\hline & & $<3$ & $2(3.7 \%)$ & $4(7.4 \%)$ & $2(4.0 \%)$ & \\
\hline & V4 & $3-7$ & $51(94.4 \%)$ & $50(92.5 \%)$ & 47 (95.9\%) & 0.59 (NS) \\
\hline & & $>7$ & $1(1.9 \%)$ & 0 & 0 & \\
\hline
\end{tabular}

Table 5: Number of bowel movement.

NS: Not Significant: $\mathrm{p}>0.05$.

\section{Discussion}

The IBS is a multifactorial disease with many therapeutic options. It is mainly geared towards IBS type: diarrhea, constipation or mixed. Various pain reliever are available such as: antispasmodics, peppermint oil, selective serotonin reuptake inhibitors (SSRIs), and tricyclic antidepressants [11,12]. Patient education and reassurance as to the benign natural history is the key for treatment [12].

In this study, most patients were females which is similar to other studies [13]. Patients with IBS present recurrent pain or discomfort for at least 3 days per month and two of the following features: amelioration with defection, change in stool consistency or frequency [14].

In our study, patients in all groups had mostly abdominal pain followed by abdominal bloating and flatulence and diarrhea.

Although we tried to randomize patients as much as possible, there was a statistical significant difference between types of IBS.

In a clinical trial assessing the efficacy of probiotics versus placebo groups, the probiotic group had a significantly lower abdominal pain score but no improvement in abdominal bloating sensation [15]. This was seen in our study at 4 months where the abdominal pain score decreased significantly in symbiotic group.

The improvement of the bloating score in the three groups was not significantly different. However, in the Vita Colon Relief ${ }^{\circledR}$ group, $1.8 \%$ of patients had scored 1 in bloating score and this number increased to $42.5 \%$ in the fourth visit (at 6 moths). Fifteen studies of 12 different probiotics evaluated the improvement of bloating/ distention in patients with IBS [16]. Seven studies proved statistically beneficial effect in patients treated with specific probiotics compared to placebo group [16].

The health related quality of life has been evaluated in two studies assessing the effect of fermented milk on patients with IBS $[17,18]$. In both studies, the fermented milk showed beneficial effect on quality of life. The latter has significantly increased after 4 weeks in patients receiving probiotics compared to placebo [18]. Similarly, for the second article, the quality of life improved after 3 - 6 weeks but the results didn't differ between placebo and probiotics groups [17]. In our study, the quality of life improved in both groups and was significantly higher in probiotics group.

Four studies assessed bowel habit and stool frequency [19-22]. No statistically significant difference was seen between symbiotic 
and placebo groups in all types of IBS [19-22]. However, this was in opposition to our study results, where a statistically significant difference was seen between the 3 groups and an improvement in stool frequency in symbiotic group.

\section{Conclusion}

In the light of current knowledge, symbiotic seems to be an efficient and tolerable treatment for IBS. It proved an ability to improve $100 \%$ the quality of life, cause an important decrease in abdominal pain score and bloating score. However, additional standardization of treatment dosing-protocols and further randomized clinical trials are needed to establish a consensus about the use of probiotics in IBS. Despite needed research, IBS symbiotic appears as a potential treatment that ease IBS symptoms.

\section{Bibliography}

1. Van den Houte K., et al. "Prevalence and impact of self-reported irritable bowel symptoms in the general population". United European Gastroenterology Journal 7 (2019): 307-315.

2. Cole JA., et al. "Migraine, fibromyalgia, and depression among people with IBS: a prevalence study". BMC Gastroenterology 6 (2006): 26.

3. AlAmeel T., et al. "The Prevalence of Irritable Bowel Syndrome Among Board-Certified Medical Doctors In Saudi Arabia: A Cross-sectional Study". Journal of the Canadian Association of Gastroenterology (2019).

4. Wilkins T., et al. "Diagnosis and management of IBS in adults". American Academy of Family Physicians 86 (2012): 419-426.

5. Preston K., et al. "Lactobacillus acidophilus CL1285, Lactobacillus casei LBC80R and Lactobacillus rhamnosus CLR2 improve quality-of-life and IBS symptoms: a double-blind, randomised, placebo-controlled study". Beneficial Microbes 9 (2018): 697706.

6. Didari T., et al. "Effectiveness of probiotics in irritable bowel syndrome: Updated systematic review with meta-analysis". World Journal of Gastroenterology 21 (2015): 3072-3084.

7. Fond G., et al. "Anxiety and depression comorbidities in irritable bowel syndrome (IBS): a systematic review and metaanalysis". European Archives of Psychiatry and Clinical Neuroscience 264 (2014): 651-660.
8. Ivana Milosevic., et al. "Gut-Liver Axis, Gut Microbiota, and Its Modulation in the Management of Liver Diseases: A Review of the Literature (2019).

9. Zamani M., et al. "Systematic review with meta-analysis: the prevalence of anxiety and depression in patients with irritable bowel syndrome". Alimentary Pharmacology and Therapeutics 50 (2019): 132-143.

10. Lacy BE and Patel NK. "Rome Criteria and a Diagnostic Approach to Irritable Bowel Syndrome". Journal of Clinical Medicine (2017): 6.

11. Shih DQ and Kwan LY. "All Roads Lead to Rome: Update on Rome III Criteria and New Treatment Options". Gastroenterology Report 1 (2007): 56-65.

12. Dale HF., et al. "Probiotics in Irritable Bowel Syndrome: An Upto-Date Systematic Review”. Nutrients (2019): 11.

13. Mearin F., et al. "Bowel Disorders". Gastroenterology (2016).

14. Haefeli M and Elfering A. "Pain assessment". European Spine Journal 15.1 (2006): S17-24.

15. Weaver KR., et al. "Irritable Bowel Syndrome". American Journal of Nursing 117 (2017): 48-55.

16. Meleine $M$ and Matricon J. "Gender-related differences in irritable bowel syndrome: potential mechanisms of sex hormones". World Journal of Gastroenterology 20 (2014): 67256743.

17. Saha L. "Irritable bowel syndrome: pathogenesis, diagnosis, treatment, and evidence-based medicine". World Journal of Gastroenterology 20 (2014): 6759-6773.

18. Zeng J., et al. "Clinical trial: effect of active lactic acid bacteria on mucosal barrier function in patients with diarrhoea-predominant irritable bowel syndrome". Alimentary Pharmacology and Therapeutics 28 (2008): 994-1002.

19. Hungin AP., et al. "Systematic review: probiotics in the management of lower gastrointestinal symptoms in clinical practice -- an evidence-based international guide". Alimentary Pharmacology and Therapeutics 38 (2013): 864-886.

20. Guyonnet D., et al. "Effect of a fermented milk containing Bifidobacterium animalis DN-173 010 on the health-related quality of life and symptoms in irritable bowel syndrome in adults 
in primary care: a multicentre, randomized, double-blind, controlled trial". Alimentary Pharmacology and Therapeutics 26 (2007): 475-486.

21. Guyonnet D., et al. "Fermented milk containing Bifidobacterium lactis DN-173 010 improves gastrointestinal well-being and digestive symptoms in women reporting minor digestive symptoms: a randomised, double-blind, parallel, controlled study". British Journal of Nutrition 102 (2009): 1654-1662.

22. Kim HJ., et al. "A randomized controlled trial of a probiotic, VSL\#3, on gut transit and symptoms in diarrhoea-predominant irritable bowel syndrome". Alimentary Pharmacology and Therapeutics 17 (2003): 895-904.

\section{Assets from publication with us}

- Prompt Acknowledgement after receiving the article

- Thorough Double blinded peer review

- Rapid Publication

- Issue of Publication Certificate

- High visibility of your Published work

Website: www.actascientific.com/

Submit Article: www.actascientific.com/submission.php

Email us: editor@actascientific.com

Contact us: +919182824667 